\title{
1 Underwater Acoustic Emission Monitoring - Experimental investigations and \\ 2 acoustic signature recognition of Synthetic Mooring Ropes
}

3

4

5

6

7

8

Imran Bashir ${ }^{1}$, Jodi Walsh ${ }^{1,2}$, Philipp R Thies ${ }^{1}$, Sam D Weller ${ }^{1}$, Philippe Blondel ${ }^{2}$, Lars Johanning ${ }^{1}$

${ }^{1}$ College of Engineering, Mathematics and Physical Sciences, Renewable Energy Research Group, University of Exeter, Penryn Campus, Treliever Road, Penryn, TR10 9FE, UK

${ }^{2}$ Department of Physics, University of Bath, Claverton Down Road, Bath, BA2 7AY, UK

\section{Abstract}

Mooring ropes are essential components of offshore installations, and synthetic ropes are increasingly preferred because of their favourable cost to weight ratios. In-service condition of these materials is traditionally monitored through costly visual inspection, which adds to the operating costs of these structures. Acoustic Emissions (AE) are widely used for condition-monitoring in air, and show great potential underwater. This paper investigates the AE signatures of synthetic mooring ropes subjected to sinusoidal tension-tension loading in a controlled environment, using a large-scale dynamic tensile test rig. With a linear array of 3 broadband $(20 \mathrm{~Hz}-50 \mathrm{kHz})$ hydrophones, four main signatures are identified: low-to high frequency, low-amplitude signals (50 Hz-10 kHz), lowamplitude broadband signals ( $10 \mathrm{kHz}-20 \mathrm{kHz})$, high amplitude signals $(10 \mathrm{~Hz}-48 \mathrm{kHz})$ and mediumamplitude signals $(500 \mathrm{~Hz}-48 \mathrm{kHz})$. These AE types are related to different stages of rope behaviour, from bedding-in to degradation and failure. The main findings are that the failure location and breaking load can be identified through the detection of AE. The occurrence of high amplitude AE bursts in relation to the applied tensile load allows the detection of an imminent failure, i.e. prior to the failure event. These initial results indicate that $A E$ analyses can enable the integrity of synthetic mooring ropes to be monitored.

Keywords: Acoustic Emissions (AE) - Mooring ropes - Wave Energy Converters (WECs) Condition Health Monitoring (CHM) - Reliability, Mooring ropes

\section{Introduction}

Most offshore structures need mooring systems, in order to provide a restoring force to counteract the effects of wind, wave and current loads. As operations move into more challenging marine environments (e.g. deeper waters or wave-energy generation), the offshore industry has repeatedly expressed concerns about the frequency of mooring line failures [1], potentially resulting in high cost mooring designs. Steel chain and wire rope have conventionally been used, but contemporary designs often feature synthetic polyester ropes which typically have a lower submerged mass per unit length, a lower cost per unit length and the potential to reduce peak loadings [2], [3]. Mooring ropes will be subject to variable loads throughout their lifetime, affecting their operational properties (i.e. stiffness and damping) and potentially inducing fatigue [4]. For the most critical assets (e.g. oil platforms), regular inspection with submersible vehicles is still the tool of choice for conditionmonitoring, despite its known limitations [1] and the latest guidelines recommend full replacement of ropes every few years [5]. Direct inspection is not easily carried out in more challenging environments, 
for example in the energetic conditions suited to Wave Energy Converters (WECs) or in the strong currents favoured for tidal turbines[6]. Mooring costs correspond to more than $10 \%$ of the capital cost of a typical WEC installation [7] and regular visual inspection with submersible vehicles would further affect the costs of marine renewable energy production, especially when scaled up to the dense arrays now planned. Some limited applicable mooring monitoring systems have been developed such as MOORASSURE, Inter-M Pulse, Load Cell Tension and Inclination Monitoring [8]. Other monitoring methods include steel catenary riser inclination/vibration, tendon tensions, fibre optic long base strain gauges, mooring winch vendor and pull tube monitoring [9]. However, the reliability of most existing monitoring techniques has not be proven and most are only capable of detecting the failure but not the degradation of the mooring lines [8], [10].

Remote monitoring of mooring condition using Acoustic Emissions ( $\mathrm{AE}$ ) is an attractive option and, it should be possible to monitor a large variety of mooring structures at once, for a much lower cost. Condition Health Monitoring has long used AE in air, for a variety of systems and application such as $A E$ monitoring of wire ropes [11], [12]. Acoustic waves propagate better in water, being less attenuated over larger distances, and recent work showed WEC signatures could be distinguished up to $200 \mathrm{~m}$ away [13]. AE from mooring ropes needs to be separated from other noises associated with device operation (e.g. the Power-Take Off system of a WEC), maintenance (e.g. supply or repair vessels) and environment (wind, weather and waves, mostly) [14]. In the case of WECs, this is exacerbated by the fact that mooring connections can significantly affect energy absorption and production [15], potentially changing the acoustic signature from surface waves. It is therefore extremely important to understand the exact acoustic contributions of mooring ropes to the soundscape, in particular as they approach failure.

This article focuses on polyester ropes, as they are potentially an enabling technology for costeffective mooring systems [3]. Polyester ropes are preferable over steel ropes as certain materials and constructions display greater compliance which can lead to a reduction in peak loadings. Their operational characteristics are however complex, often with time-dependent viscoelastic and viscoplastic behaviour [16]. For the purpose of this study three samples of a typical rope material and construction were tested in the controlled environment of the large-scale dynamic test rig DMaC (Dynamic Marine Component, University of Exeter), under a variety of loads typical of marine operations (Section 2). Their Acoustic Emissions were monitored with 3 broadband hydrophones and specific signatures were identified in spectrograms (Section 3). The time-of-arrival localisation of specific $A E$ is linked to the physical processes of degradation and failure (Section 4). This Section also identifies which characteristics can best be used at sea, focusing on the application of this technique to Wave Energy Converter mooring system monitoring. The concluding remarks are presented in Section 5.

\section{Experimental Testing}

Underwater acoustic testing has been carried out to study the AE of synthetic fibre mooring ropes. The aim of the testing was to detect the release of acoustic waves or energy in response to applied loading regimes, informing remote monitoring options for reliability and durability assessment of polyester ropes. 


\subsection{Samples}

The rope type chosen for the experiments was a 12-strand double-braid polyester rope with a nominal diameter of $24 \mathrm{~mm}$. The rope has six right-hand laid strands and six left-hand laid strands that produce a torque balanced rope. It is a double-braided rope with a core enclosed by an outer braid cover. The internal and external core construction are both laid in a braided assembly. This $12-$ strand double-braid rope construction offers high strength and very good abrasion resistance and as such is well suited to MRE mooring applications [3].

Acoustic testing was carried out on three polyester rope samples from the same manufacturers batch, referred to as R1, R2 and R3 in the following sections. The three samples were eye-spliced in order to connect them into the test rig using mooring shackles. The total eye-to-eye length of the three spliced ropes before loading was measured to be R1 $=3.53 \mathrm{~m}, \mathrm{R} 2=3.60 \mathrm{~m}, \mathrm{R} 3=$ $3.62 \mathrm{~m}$. The rope sample properties are given in Table I as stated by the manufacturer [17]. Figure 1 (a) provides a schematic of the construction of double braided rope and Figure 1(b) shows the photograph for internal core and outer cover of the rope.

Table I Rope properties \& Specification [17]

\begin{tabular}{|l|l|}
\hline Material & High tenacity Polyester Multifilament fibre \\
\hline Construction & 12 strands double braid \\
\hline Nominal diameter & $24 \mathrm{~mm}$ \\
\hline Nominal mass in water & $0.13 \mathrm{~kg} / \mathrm{m}$ \\
\hline Minimum breaking force & $129 \mathrm{kN}$ \\
\hline
\end{tabular}

(a)

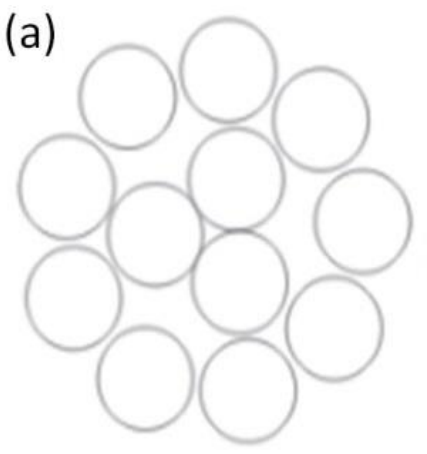

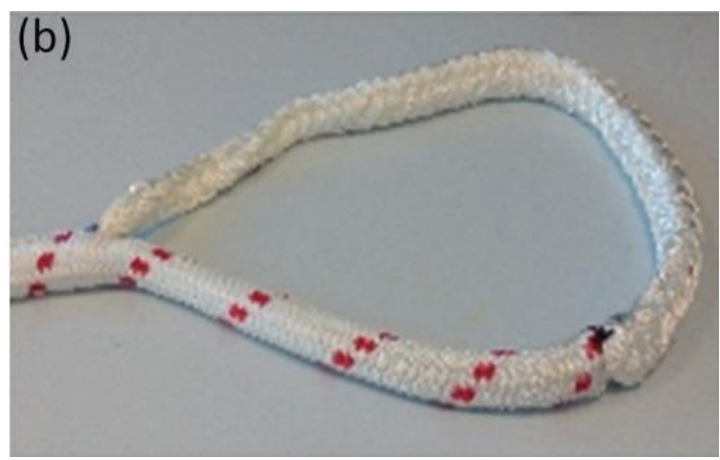

Figure 1: a. Construction of the 12 strand double-braid polyester rope sample. b. Photograph of the core and cover of the rope during the eye splicing process.

\subsection{Test facility and Tensile load profile}

The DMaC facility is a purpose built test rig that can replicate the forces and motions that components are subjected to in offshore applications. The rig can test component specimens of up to 6 meters in length and has the capability of carrying out immersed component testing. The linear actuator and the headstock allow the dynamic testing of large scale components in a fully-controlled environment by applying realistic motion and load time-series [18].

All three rope samples were subjected to similar tensile cyclic loading regimes with the objective to progressively increase the maximum load until failure. Before applying tensile cyclic loading, bedding-in was carried out for all three rope samples. The bedding-in procedure was specified using the rope $\mathrm{MBL}$ as outlined in [16]. However, due to time constraints a shortened procedure was 
specified with shorter load-hold durations. A twenty minute bedding-in time interval comprising hold and ramp cycles lasting twenty seconds with a minimum and maximum load of $5 \mathrm{kN}$ and $20 \mathrm{kN}$ respectively was used. The time series plot for bedding-in cycles is given in Figure 2 (a). It is acknowledged that the samples may not have been completely bedded-in after this process.

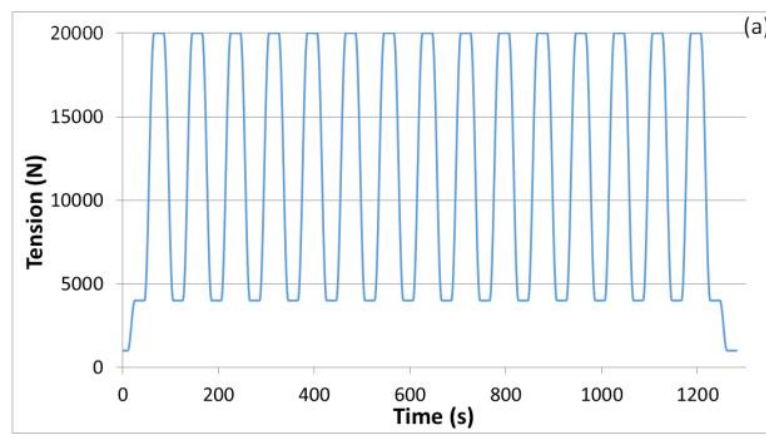

(a)

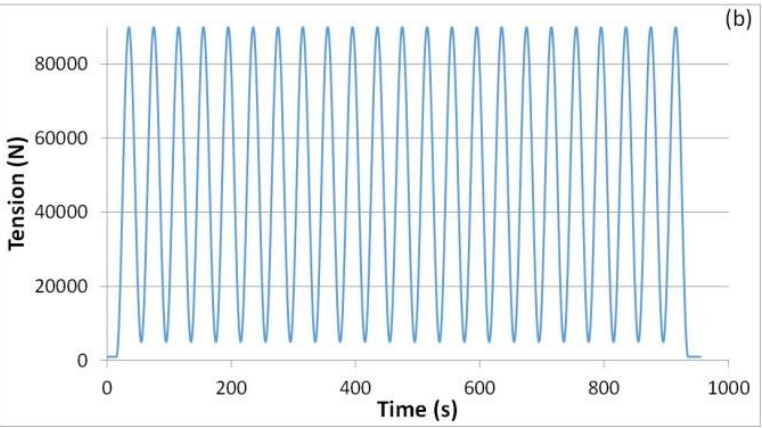

Figure 2: a. Twenty minute bedding-in time schedule for rope, 20 second hold and ramping, Min load = $5 \mathrm{kN}, \mathrm{Max}$ load $=20$ $k N(b)$ Fifteen minute cyclic loading time schedule, Min load = $5 \mathrm{kN}$, Max load = $90 \mathrm{kN}$ (an example plot as maximum load progressively increased until failure).

The rope samples were subjected to sinusoidal load cycles, oscillating between the minimum and maximum loads indicated. The minimum loading was set to $5 \mathrm{kN}$, whilst the maximum loading was stepwise increased from $30 \mathrm{kN}$ until rope failure. An example time series plot for cyclic loading of between $5 \mathrm{kN}$ and $90 \mathrm{kN}$ is shown in Figure 2 (b). The cyclic loading was increased linearly in order to study the acoustic emission for all regimes. Rope sample R1 was tested with slightly larger step-sizes to identify loads of increased acoustic release. Rope samples R2 and R3 were tested with smaller incremental steps to provide a different load increment. Initially, the rope sample R1 was subjected to load cycles with a time period of $40 \mathrm{~s}$, and this was later increased to $60 \mathrm{~s}$ for rope sample R2 and R3 to minimize the background noise caused by the test rig. Table II summarizes the individual test cycles experienced by each rope sample.

Table II Loading regime and time schedule for cyclic loading.

\begin{tabular}{|c|c|c|c|c|c|c|c|c|c|c|c|c|c|c|}
\hline \multirow{2}{*}{ 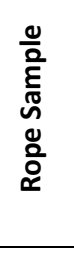 } & \multirow{2}{*}{$\begin{array}{l}\frac{\tilde{d}}{0} \\
\frac{0}{0} \\
\dot{0} \\
\frac{\dot{z}}{\pi} \\
\dot{0} \\
1\end{array}$} & \multirow{2}{*}{ 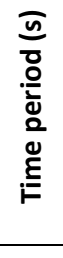 } & \multirow{2}{*}{ 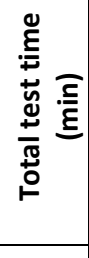 } & \multirow{2}{*}{ 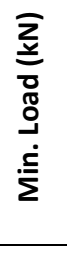 } & \multicolumn{10}{|c|}{$\begin{array}{l}\text { Maximum Load (kN) 'Load cycles, oscillating between the } \\
\text { minimum }(5 \mathrm{kN}) \text { and maximum loading as given (See Figure } 2(\mathrm{~b}))^{\prime}\end{array}$} \\
\hline & & & & & & \multicolumn{9}{|c|}{$\begin{array}{l}\text { Diagonal line indicates that only rope sample R3 } \\
\text { experienced those loading cycles as it failed at higher load }\end{array}$} \\
\hline \multirow{2}{*}{ R1 } & \multirow{2}{*}{22} & \multirow{2}{*}{40} & \multirow{2}{*}{15} & \multirow{2}{*}{5} & 30 & 40 & 50 & 60 & 65 & 70 & 75 & 80 & 82.5 & 85 \\
\hline & & & & & 90 & 91 & 92 & 93 & 94 & 95 & 96 & 97 & 98 & \\
\hline \multirow{6}{*}{$\begin{array}{l}\text { R2/ } \\
\text { R3 }\end{array}$} & \multirow{6}{*}{20} & \multirow{6}{*}{60} & \multirow{6}{*}{20} & \multirow{6}{*}{5} & 30 & 40 & 40 & 50 & 60 & 62.5 & 65 & 67.5 & 70 & 71 \\
\hline & & & & & 72 & 73 & 74 & 75 & 76 & 77 & 78 & 79 & 80 & 81 \\
\hline & & & & & 82 & 83 & 84 & 85 & 86 & 87 & 88 & 89 & 90 & 90.5 \\
\hline & & & & & 91 & 91.5 & 92 & 92.5 & 93 & 93.5 & 94 & 94.5 & 95 & 95.5 \\
\hline & & & & & 96 & 96.5 & 97 & 97.5 & 98 & 100 & 102 & 104 & 105 & 106 \\
\hline & & & & & 108 & 110 & 112 & 112 & & & & & & \\
\hline
\end{tabular}




\section{Acoustic set up}

In order to carry out underwater acoustic testing of polyester ropes, a linear array consisting of three hydrophones was installed inside the DMaC test rig. Two of the sensors were SQ26-08 Cetacean cylindrical shaped directional hydrophones and the third was a ball-shaped JS-B100-C4DSPA Integrated Acoustic Sensor. Table III summarizes the specifications for both types of hydrophones used. The two cylindrical hydrophones were placed at the two ends of the rope samples close to the splices ('Headstock hydrophone' and 'Z-ram hydrophone') and the third ball hydrophone was placed at the centre of the rope samples ('Centre hydrophone'). The hydrophones were placed at equal distances (i.e. $1.6 \mathrm{~m}$ ) along the rope in order to cover the entire length of the rope. A schematic of this configuration and photographs of the mounted hydrophones are shown in Figure 3.

The test rig was filled with fresh water and the rope samples were submerged $10 \mathrm{~cm}$ deep. The hydrophone array was placed at a distance of $10 \mathrm{~cm}$ next to the length of the rope and at the same depth in the water. The hydrophones were enclosed in a wire cage to protect them from damage. Similarly, the cables of the hydrophones were passed through PVC pipes for protection. The pipes were filled with self-expanding foam to avoid them acting as acoustic wave-guides. The hydrophones were fixed to the rig using G-clamps and timber with the use of protective padding to avoid the transmission of any external vibration.

Table III Specification for hydrophones used for measurements.

\begin{tabular}{|l|l|l|}
\hline Hydrophone type & $\begin{array}{l}\text { Frequency Range } \\
(\mathrm{kHz})\end{array}$ & $\begin{array}{l}\text { Transducer Sensitivity } \\
(\mathrm{dB}, \text { re 1V/ } \mu \mathrm{Pa})\end{array}$ \\
\hline SQ26-08 Cetacean & $0.02-50$ & -169 \\
\hline JS-B100-C4DS-PA & $0.02-50$ & -168 \\
\hline
\end{tabular}
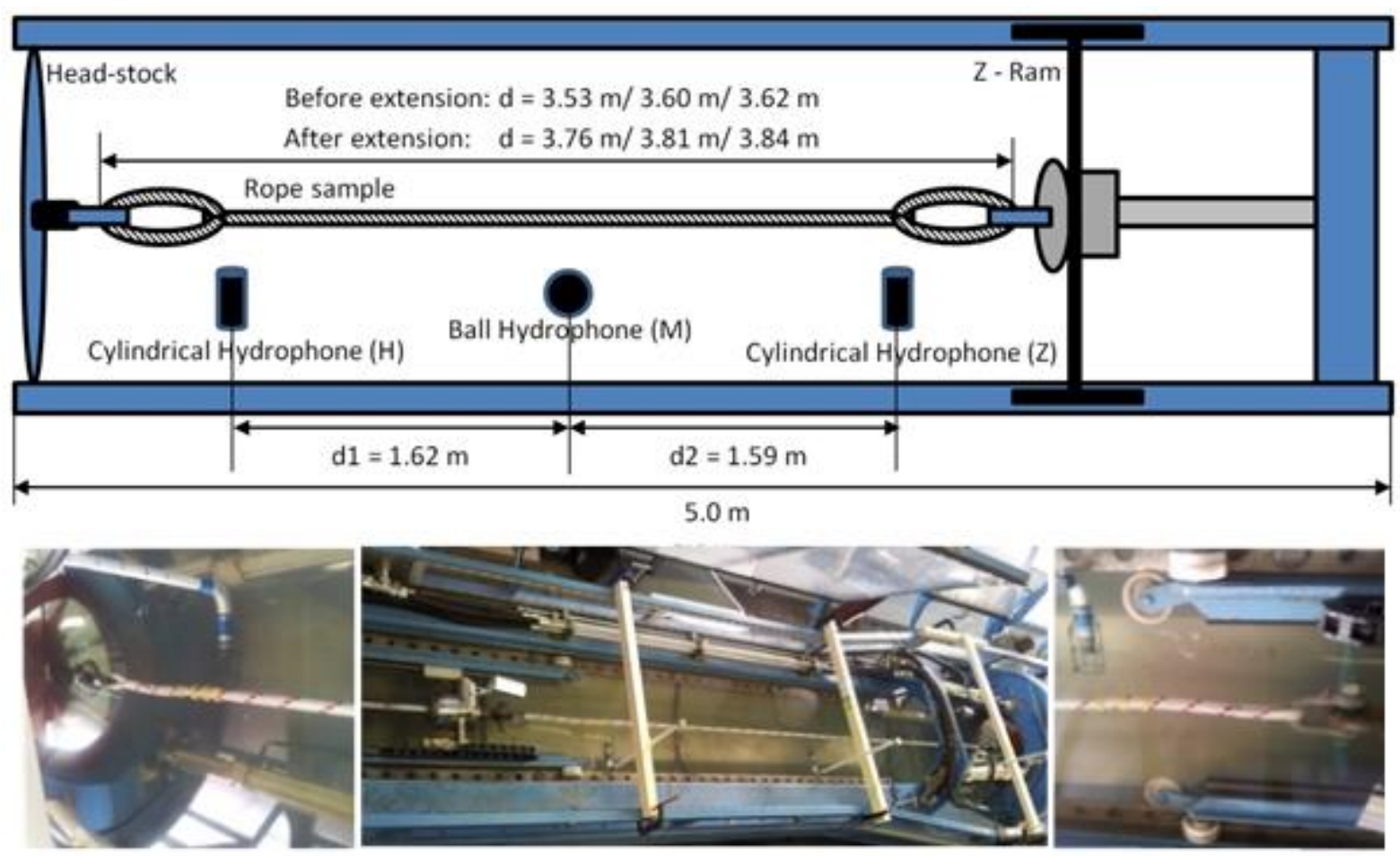

Figure 3: Schematic diagram (top view) and associated photographs showing the experimental set up for underwater acoustic rope testing inside the DMaC test rig. 


\subsection{Limitations}

Overall, the designed setup provides a suitable method for testing ropes. However, it is acknowledged that there are some limitations to the experimental method if compared to the AE that would be measured offshore. The selected ropes are of small diameter and short in length compared to mooring lines in sea. These experiments are carried out by shortening the length of the rope as it is not possible to test full length ropes in most tension-tension test rigs and furthermore it is standard practice to test short samples [3]. The mooring ropes used in the test are of similar material and construction, therefore the test results are deemed to be representative. Similarly, the loading has been carried out using accelerated testing with the assumption that the damage accumulates over the lifetime of the ropes [19]. The correlation between the accelerated rope testing for synthetic ropes under controlled laboratory conditions (DMaC test rig) has been compared with real sea data [20]. The comparison between two tests and numerical simulation concluded that it might be possible to carry out accelerated testing on ropes by accumulating failures modes [20]. In this study the number of samples are limited; however, all samples produce very consistent and similar results. The work will be extended to more samples as well as field testing.

\subsection{Data analysis methods}

Most of the AE signals are non-stationary and often comprise overlapping transients whose waveforms and arrival time are unknown. Therefore, instantaneous and non-averaged frequency analysis was used for feature extraction, which can be obtained using Short Time Fourier Transform (STFT) [21], [22]. Figure 4 (a) shows the schematic for STFT to obtain spectrogram. Figure 4 (b) provides an example plot for time domain data and corresponding spectrogram. By using STFT, the instantaneous acoustic features in time domain data (i.e. peaks) are clearly distinct in the spectrogram (i.e. transitions in spectral contents). The STFT data analysis technique is limited by its fixed time and frequency resolution i.e. a narrower window gives good time but poor frequency resolution and vice versa. Poor resolution may contribute to a loss of possible AE features; therefore multiple windowing widths (256, 512, 1024, 2048, 4096, 6400 and 8192 data points), filtering and overlap were used for data analysis to resolve this issue.

The acoustic sources can be localised by calculating the time difference of arrival measured with the associated pairs of hydrophones. The time difference between two signal arrivals can be calculated using the cross-correlation function [22], which is a measure of similarity between two waveforms as a function of time-lag applied to one of them.
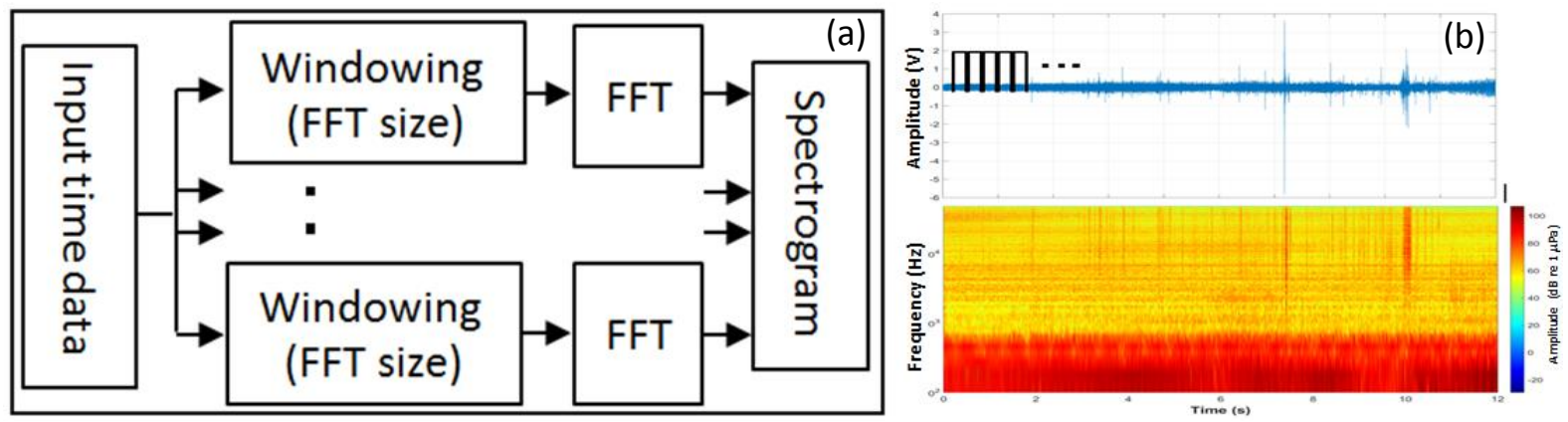

Figure 4: (a) Schematic diagram show STFT analysis technique (b) An example time domain signal and corresponding spectrogram output. 


\section{Results}

This section presents the key results obtained from the experiments outlined in the last section. Firstly, the background noise of the test rig is characterised in order to isolate the acoustic emissions from the rope specimens. Secondly, the observed acoustic emission signals are classified. Finally, the key observations for all three rope samples are summarised.

\subsection{Background noise}

The hydraulic test rig produces noise stemming from the hydraulic pumps, valve activity and mechanical movements. This background noise can potentially mask the AE signal from the rope specimens, and it was thus important to characterise these signals. The noise characterisation of the test rig was carried out by filling it with water and monitoring the AE signal during different cyclic loading conditions in force control mode.

Figure 5 (a) shows the time domain recording of the linear actuator hydrophone (red line); it has been superimposed with the loading cycle of the test rig (blue line). The amplitude of recorded noise and loading cycles has been normalised to allow a direct comparison. It can be observed that the level of background noise is governed by the motion of the hydraulic actuator. Increased noise amplitudes are recorded when the actuator is moving, i.e. ramping up or down towards maximum or minimum loading. Noise levels are reduced when the loading reaches a maximum - i.e. when the linear actuator is relatively steady. Knowledge of this acoustic behaviour allows distinction to be made between the noise produced by the DMaC facility and samples, particularly at higher loadings.

The test rig produced a continuous high amplitude and low frequency tonal noise at $230 \mathrm{~Hz}$. The harmonics of the tonal noise can be seen in the spectrogram along with high frequency cracking/mechanical noises due to valves and movement of the linear actuator as shown in Figure 5 (b). The headstock of the rig was held at a fixed position; therefore, the source of noise was due to the movement of the linear actuator alone.

Furthermore, the $A E$ of the test rig is very periodic, which improves the predictability of this noise source. The amplitude of the noise produced varies in accordance with the time period of the loading cycle, i.e. it depends on the speed of linear actuator movement. The optimum loading cycle was found to be at $60 \mathrm{~s}$ duration where the linear actuator produces minimum noise for a given load. Thus longer cycle durations were selected to reduce the $A E$ emissions from the test rig. It is acknowledged here that the $60 \mathrm{~s}$ duration load cycle is larger than what would be experienced by mooring systems of small wave energy converters excited at first-order wave frequencies. 

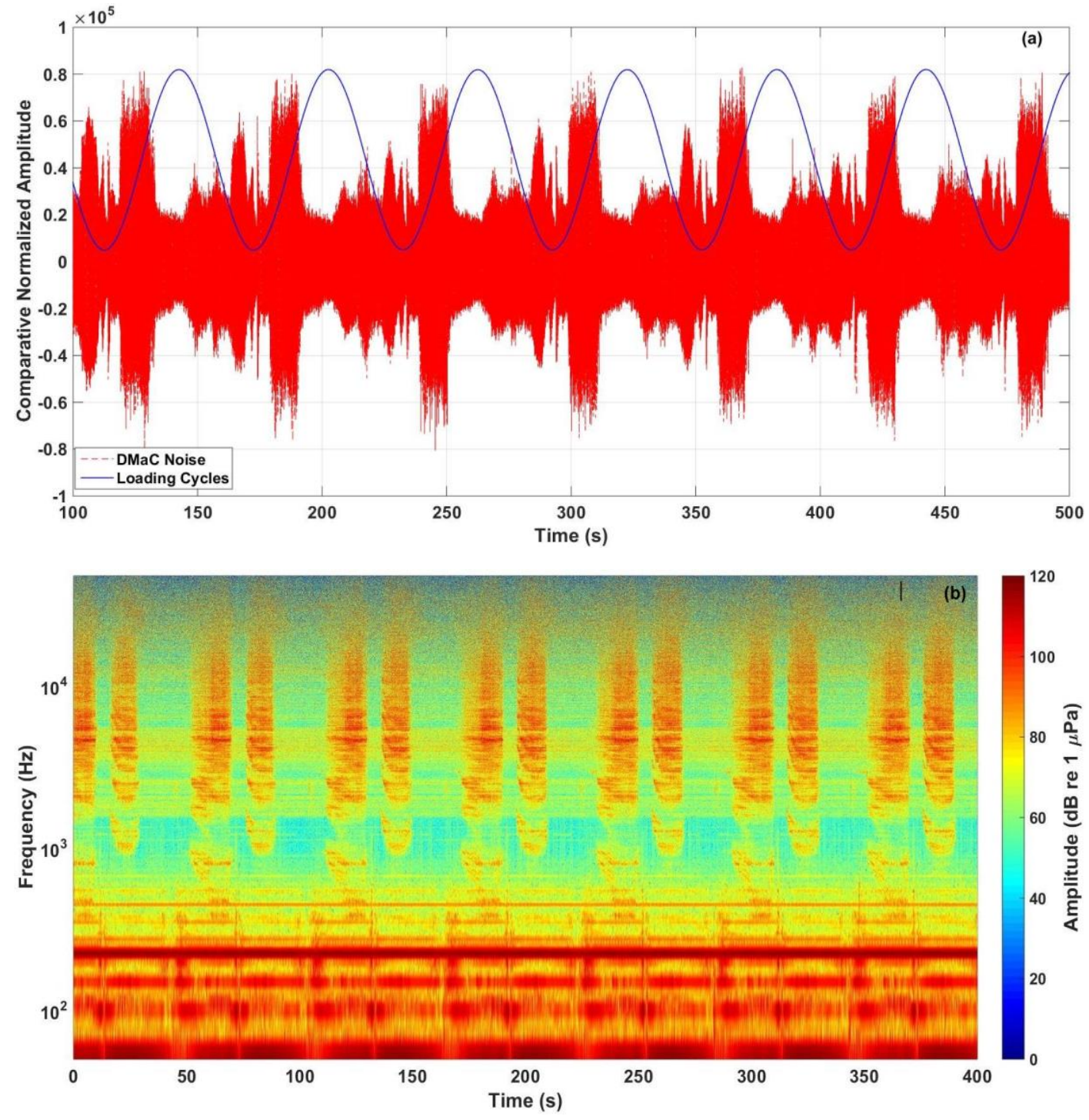

Figure 5: a. Time domain recording for the hydrophone placed close to linear actuator (Z-ram, red line), with superimposed loading cycles (blue line); the amplitudes of the recorded data and the loading cycles have been normalized for comparison b. Spectrogram plot.

\subsection{Acoustic emission signatures}

The polyester rope samples subjected to cyclic loading produced a variety of AE. All of the AE signatures detected from the rope specimens were bursts of sound lasting for a very short period of time in the order of $0.5 \mathrm{~ms}$, which are henceforth referred to as "signals". Impulsive signals are distinct acoustic signals separate in time while continuous signals contain a combination of indistinguishable individual waveforms. During testing a number of different signals were detected.

Figure 6 (a) shows the time domain plot for a low to high frequency acoustic signal and Figure 6 (b) gives the corresponding spectrogram. This acoustic signal spans from $50 \mathrm{~Hz}-10 \mathrm{kHz}$ and appears for very short periods of time. The measured amplitude for these signals was between 90 and $100 \mathrm{~dB}$ re $1 \mu \mathrm{Pa}$. They are few in number, typically one or two signals were detected for each rope sample studied. 

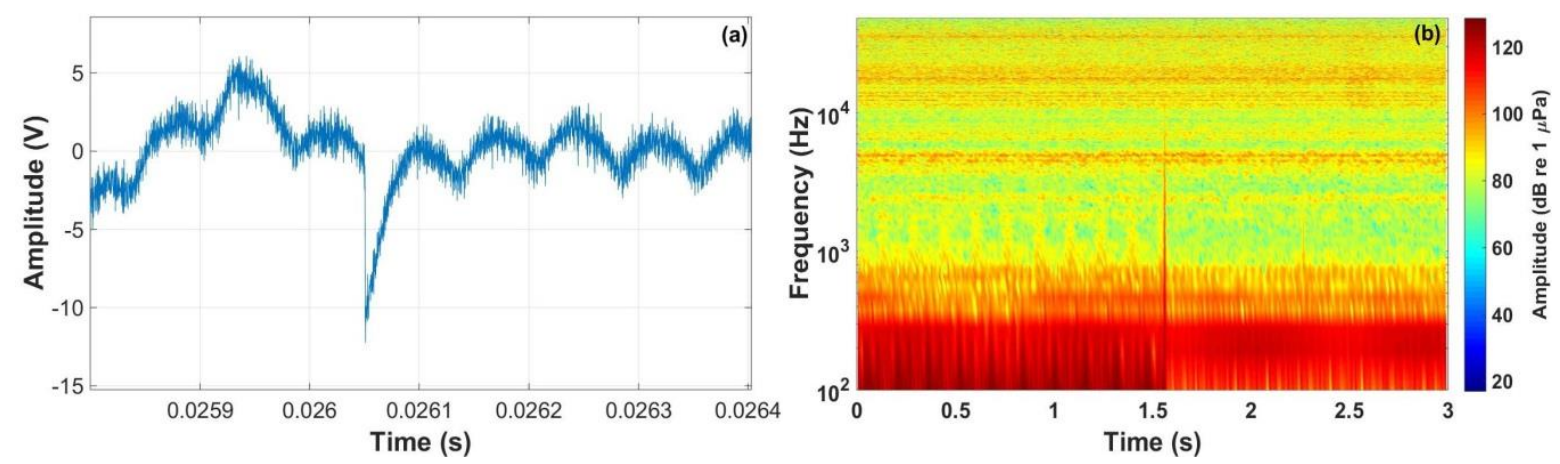

Figure 6: A representative example of a low-to-high frequency signal (a) time domain (b) spectrogram.

Figure 7 (a) shows the time domain plot for a low amplitude acoustic signal and Figure 7 (b) gives the corresponding spectrogram. The acoustic signature for the low amplitude signals is fairly narrowband as compared to the other acoustic features described later. The signature appears within the frequency range of $10 \mathrm{kHz}-20 \mathrm{kHz}$. The measured amplitude for these signals was around $90 \mathrm{~dB}$ re $1 \mu \mathrm{Pa}$. The observed acoustic signatures for the low amplitude signals were very consistent in all three ropes and produced more or less an identical signature.
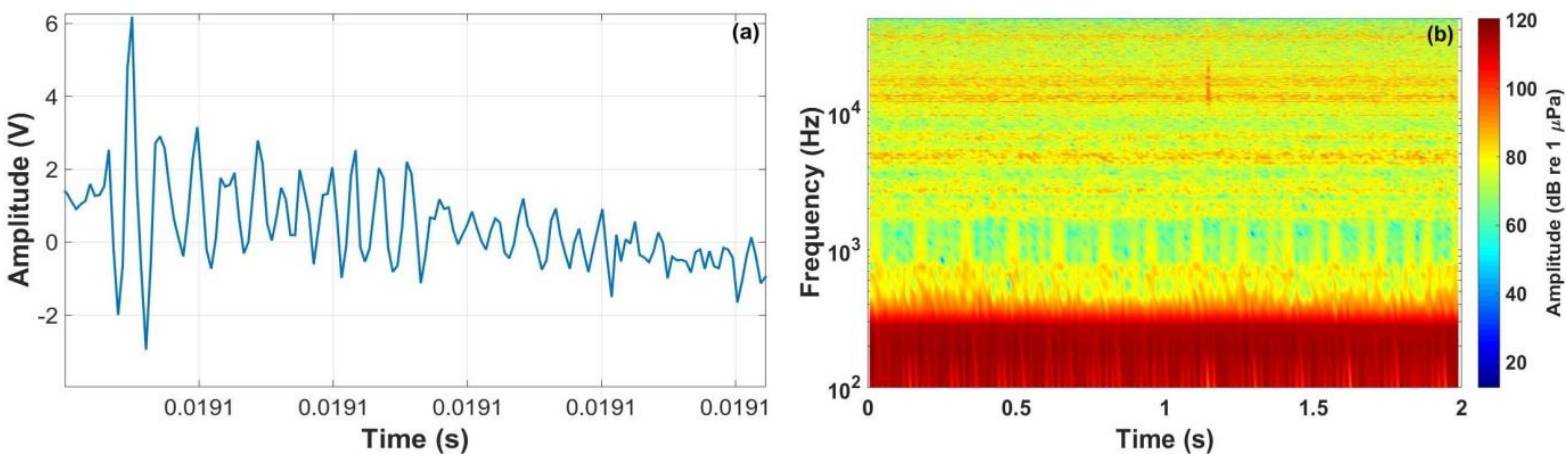

Figure 7: A representative example of a low-amplitude signal (a) time domain (b) spectrogram.

The medium amplitude signal is broadband and covers the frequency band $500 \mathrm{~Hz}-48 \mathrm{kHz}$. Time-domain and spectrogram representations of a typical medium signal are shown in Figure 8 (a) and (b). The measured amplitude for these signals was between 110 and $120 \mathrm{~dB}$ re $1 \mu \mathrm{Pa}$.
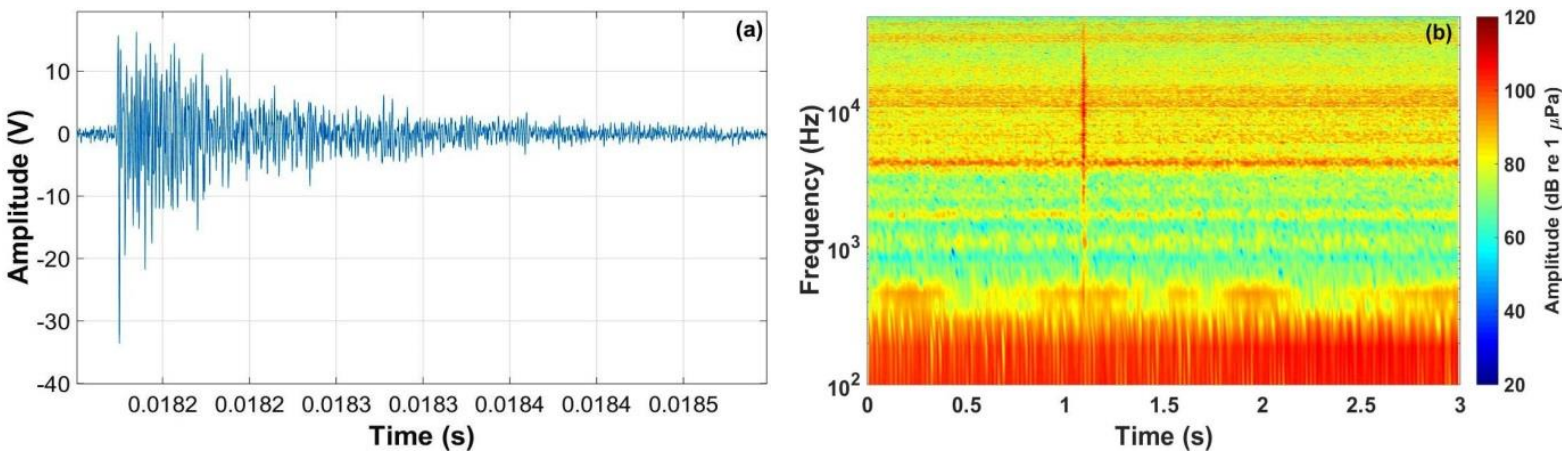

Figure 8: A representative example of a medium-amplitude signal (a) time domain (b) spectrogram.

Figure 9 (a) shows the time domain plot for a high-amplitude acoustic signal and Figure 9 (b) gives the corresponding spectrogram. The high-amplitude signal spans the entire frequency range measured, i.e. $10 \mathrm{~Hz}-48 \mathrm{kHz}$ as the hydrophone's sampling frequency was set to be $96 \mathrm{kHz}$. The spectral contents and time domain waveforms of the large $A E$ signal are identical to what was observed in all rope samples. The measured amplitude for high amplitude signals was between 120 
and $130 \mathrm{~dB}$ re $1 \mu \mathrm{Pa}$. The time domain waveform of high amplitude signals show multiple hits (i.e. each peak is counted as one hit). An average of up to thirty hit counts has been found in a high amplitude AE signal.
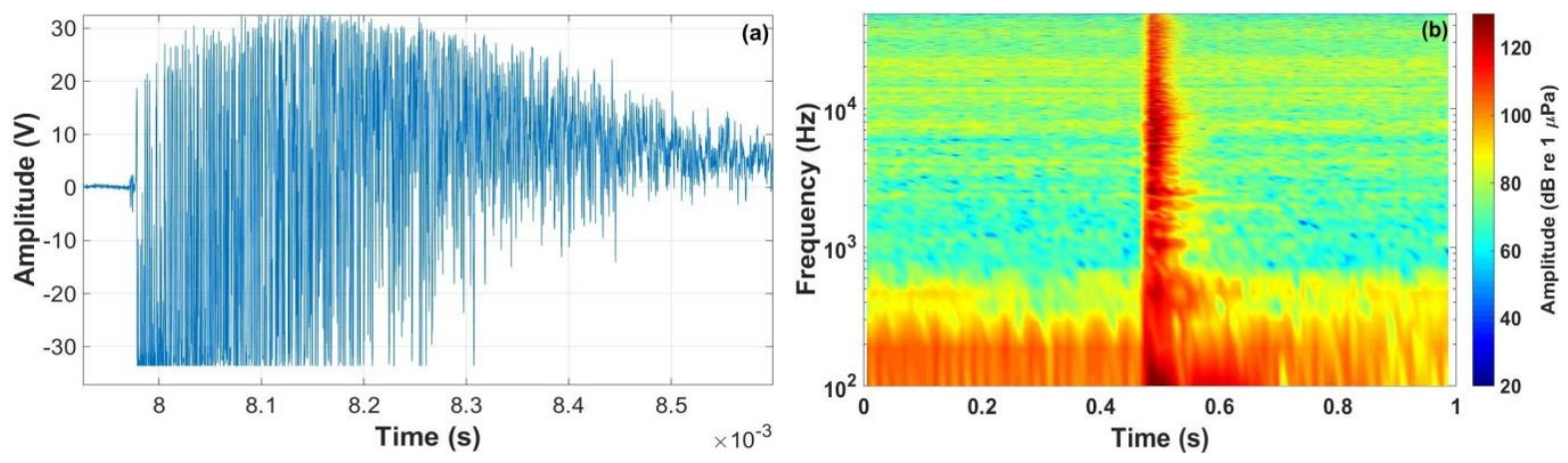

Figure 9: A representative example of a high-amplitude signal (a) time domain (b) spectrogram.

\subsection{Classification}

During testing a number of different signals were detected and hence the introduction of some descriptive language will help to classify them (Table IV).

Table IV Classification of AE signatures due to loading on polyester rope in the DMaC acoustic testing.

\begin{tabular}{|l|l|l|l|l|}
\hline Classification & $\begin{array}{l}\text { Amplitude } \\
\text { (qualitative) }\end{array}$ & $\begin{array}{l}\text { Amplitude } \\
\text { (quantitative) } \\
\mathbf{d B} \text { re } \mathbf{~} \boldsymbol{\mu P a}\end{array}$ & $\begin{array}{l}\text { Frequency } \\
\text { range } \\
\text { (kHz) }\end{array}$ & $\begin{array}{l}\text { Example } \\
\text { spectrogram }\end{array}$ \\
\hline $\begin{array}{l}\text { Low to high frequency } \\
\text { signal }\end{array}$ & Low & 100 & $0.05-10$ & Fig 6 \\
\hline Low amplitude signal & Low & 90 & $10-20$ & Fig 7 \\
\hline Medium amplitude signal & Medium & 110 & $0.5-48$ & Fig 8 \\
\hline High amplitude signal & High & 125 & $0.01-48$ & Fig 9 \\
\hline
\end{tabular}

\subsection{Full testing cycle results}

$\mathrm{AE}$ in synthetic ropes was detected as low amplitude signals when the cyclic loading was increased to more than $50 \% \mathrm{MBL}(64.5 \mathrm{kN})$. As the loading increased beyond $70 \% \mathrm{MBL}(90.3 \mathrm{kN})$, the rope samples entered into a new $A E$ regime and started producing more frequent high-amplitude $A E$ signals. Figure 10 shows the total number of $A E$ signals recorded against the maximum applied loading force for rope sample R3. 


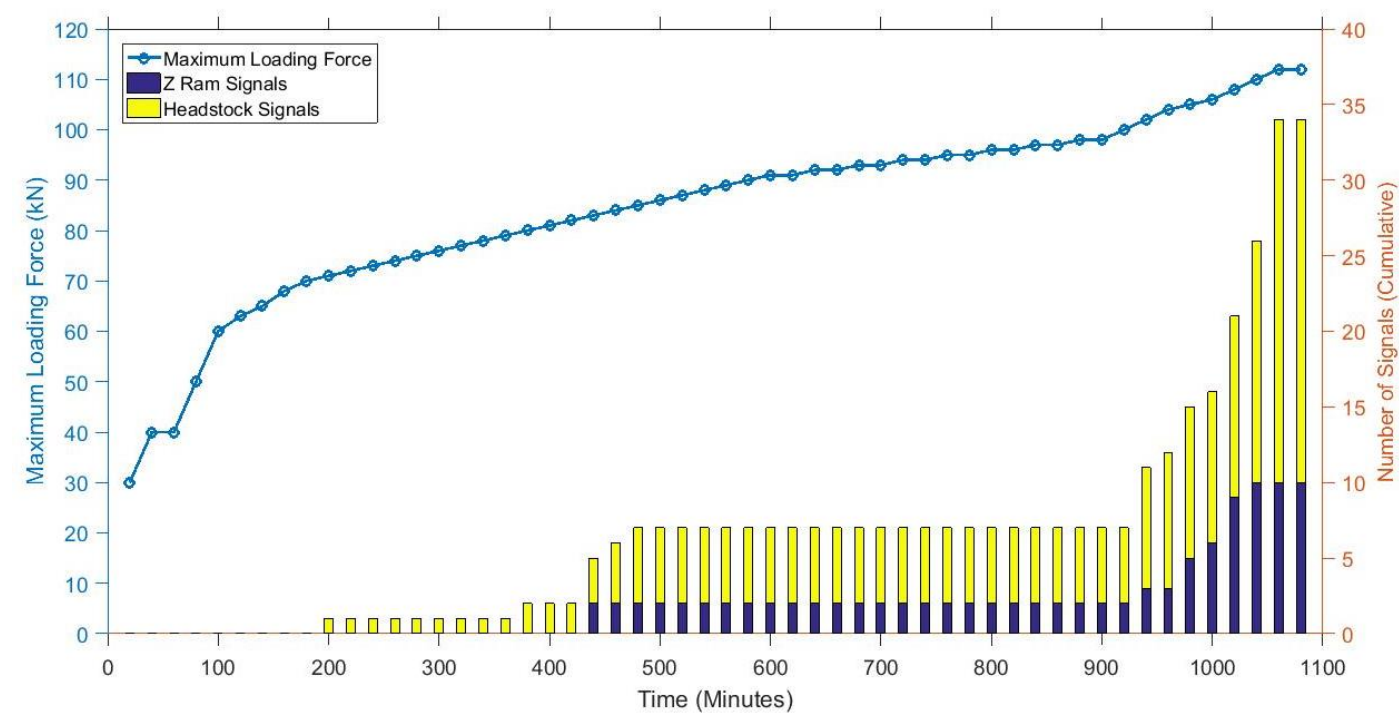

Figure 10: Total number of AE signals vs maximum loading force $(k N)$ with respect to cyclic loading (minutes) applied on rope sample $R 3$.

With the increase in loading force, the rope samples produced a series of high-frequency $A E$ signals. As the mean load was increased more high-frequency noise along with a series of large signals were produced followed by internal core and subsequent outer core failure. All rope samples failed before the rope was loaded to the MBL specified by the manufacturer i.e. the rope sample R1, R2 and R3 failed at $76 \% \mathrm{MBL}, 77 \% \mathrm{MBL}$ and $87 \% \mathrm{MBL}$ respectively. Failure location was identified by the time difference of arrival measured with pairs of hydrophones. Table $V$ summarizes the location and measured breaking load for the three rope samples. Figure 10 shows the accumulative AE signals observed under cyclic loading (rope sample R3). Under cyclic loading low-amplitude signals were recorded near the headstock. The number of signals increased leading to appearance of high amplitude signals. Similar, signals were also observed the other end of the rope (Z-ram). However, the rope failed near the headstock which shows earlier and lower amplitude signals. Therefore, it can be concluded that low-amplitude signals provide an indication of the initiation of a weak point in the rope. Similar behaviour has been observed in other two rope samples.

Table V A summary of the failure information for the 3 synthetic fibre rope samples.

\begin{tabular}{|l|l|l|l|}
\hline Sample & Rope \# R1 & Rope \# R2 & Rope \# R3 \\
\hline Actual Breaking Load (ABL) & $98 \mathrm{kN}(76 \% \mathrm{MBL})$ & $98.5 \mathrm{kN}(77 \% \mathrm{MBL})$ & $112 \mathrm{kN}(87 \% \mathrm{MBL})$ \\
\hline Failure location & Near linear actuator & Near headstock & Near headstock \\
\hline Failure images & ( & & \\
& & &
\end{tabular}

\section{Discussion}

Underwater AE measured during loading of mooring ropes has been studied at the DMaC test facility. Multiple AE signatures were recorded from the samples tested. The measurements obtained indicate that the AE signals could be related to different physical phenomena such as bedding in, slippage and 
failure. Initially low amplitude signals were detected which might be produced due to realignment or rubbing of the fibre threads in the rope [4]. Previous work focused on several different kinds of damage mechanisms in ropes and studied the performance and durability of a rope deployed for 18 months at sea [4]. The study reasoned that wear occurring due to friction between the moving fibres or yarns, accelerated by the ingress of particles into the rope structure, was a likely cause of altered rope properties including a lower measured MBL. Friction occurring between the fibres will cause localised heating of the rope and could cause AE. The low-amplitude signals recorded provide some indication of the possible initialization of weak points in the rope as all ropes failed in the proximity of where these signals had been detected first.

With the progressive application of load cycles, the rope samples started producing high-amplitude signals. The spectral contents and time domain waveforms of the high amplitude AE signals are identical to what was observed for all rope samples. Therefore, it is likely that a similar physical phenomenon is producing these signals. The hypothesis is that the high-amplitude AE signals might have been generated by the failure of load bearing elements in the rope (i.e. fibres, yarns and/or yarn assemblies) possibly caused by abrasion between contacting elements (as reported in [4]). These failure might also be the result of unequal load sharing in short rope sample. Therefore, the rope failed on either end near the splice. An average of thirty hit counts has been identified.

The time of arrival for these high amplitude signals at the hydrophones was used to locate the weak point in the rope. It was concluded that all observed high amplitude signals were more or less originating from single or multiple weak points identified earlier in the rope. Counting the number or the intensity of high amplitude signals could be used to monitor the condition of mooring ropes insitu. AE can be potentially used to predict the imminent rope failure to avoid the catastrophic incident.

As shown in Table $V$ all rope samples failed at different breaking loads at, or close to a splice. Figure 10 plots the accumulative AE signals for rope sample 3 . The other two rope samples show more or less similar trends in generation of low amplitude and high amplitude signals. This work acts as a baseline and there is a clear need to carry out testing for identical samples under identical loading conditions. Such experimental data could then be used to develop an empirical derivation for continuous monitoring and the prediction of imminent mooring rope failures.

The AE signals were produced over various frequency bands with varying amplitude. Table 2 summarises the frequency ranges and corresponding amplitudes. For AE monitoring, it is important to understand how far away the AE signals can be detected. The measured amplitudes for the lowand high-amplitude signals were around $90 \mathrm{~dB}$ re $1 \mu \mathrm{Pa}$ and $125 \mathrm{~dB}$ re $1 \mu \mathrm{Pa}$ respectively. These amplitudes can be regarded as source amplitudes, measured at a distance of $0.1 \mathrm{~m}$ from the source in a controlled laboratory environment. Ignoring other factors for transmission loss geometrical spreading $\left(15 \times \log _{10}\right.$ (distance) $)$ can be used to approximate sound attenuation over a distance from the source. The geometrical spreading transmission loss for a distance of $200 \mathrm{~m}, 500 \mathrm{~m}$ and $1 \mathrm{~km}$ is 35 $\mathrm{dB}, 41 \mathrm{~dB}$ and $45 \mathrm{~dB}$ respectively. The sound attenuation in sea is also dependent on the frequency of its propagation. The absorption due to seawater at $1 \mathrm{kHz}, 10 \mathrm{kHz}$ and $50 \mathrm{kHz}$ is $0.06 \mathrm{~dB} / \mathrm{km}, 0.76 \mathrm{~dB} / \mathrm{km}$ and $12.77 \mathrm{~dB} / \mathrm{km}$ [23]. Background noise in the ocean is usually high, with low frequencies dominated by shipping noise and higher frequencies with wave and wind noise [14]. High frequencies also experience more attenuation; therefore an AE signal with a broadband frequency spectrum is more likely to be detectable. 
The existing monitoring methods for mooring lines have limited applications. The most commonly method is visual inspection, which is challenging and potentially hazardous for divers, and also damage can occur to the mooring lines. The accumulation of marine growth can also restrict the effectiveness of visual inspections. Direct $\&$ indirect in-line tension monitoring technology exists to detect failures. Similarly, other techniques include inclinometers which detect failures through mooring line angle, load cells detect through load monitoring and GPS systems through differential displacement of mooring ropes. All these methods are capable of only detecting an already failed mooring system. The proposed technique in this paper points towards a technique that allows the continuous monitoring of mooring ropes.

Initial work on $\mathrm{AE}$ due to synthetic fibre mooring ropes shows promising results. AE monitoring can provide a multi-purpose non-invasive system which can be placed at some distance from the dynamic mooring ropes and potentially able to simultaneously monitor multiple lines in array layouts.

\section{Conclusion}

Polyester ropes are an important part of modern mooring systems. A cost-effective AE monitoring system is much needed to continuously monitor the integrity of ropes. In this study 12-strand doublebraided mooring ropes were tested in a controlled laboratory environment. At DMaC test facility, the rope samples were subjected to tensile cyclic loading regimes. The load was progressively increased until the samples failed. A linear array of three hydrophones was used to acoustically monitor the rope samples. The noise from test rig was characterized and it was found that it produces low frequency tonal at $230 \mathrm{~Hz}$, which is unlikely to effect the AE testing as mooring ropes generate AE signatures over a broad frequency band.

The $A E$ testing of mooring ropes revealed multiple types of $A E$ signals with different acoustic signatures. The AE signals have been divided into four different categories: low-amplitude signal, lowto-high-frequency signal, medium-amplitude signal and high-amplitude signal. The observed amplitudes for these signals were $90,100,110$ and $125 \mathrm{~dB}$ re $1 \mu$ Pa respectively. Similarly, the measured frequency bands for these signals were $10-20 \mathrm{kHz}, 0.05-10 \mathrm{kHz}, 0.5-48 \mathrm{kHz}$ and $0.01-$ $48 \mathrm{kHz}$ respectively. These $\mathrm{AE}$ signals are related to multiple physical processes such as slippage and failure. The time of arrival of these AE signals can be used to locate the weak point in the ropes. It is concluded that $\mathrm{AE}$ monitoring can be used to potentially predict the location of failure as well as imminent failures. The acoustic features observed in controlled laboratory environment are surprisingly consistent.

This study has demonstrated that it is in principle feasible to detect mooring line failures with acoustic emission monitoring techniques. Further work will be dedicated to examine the physical failure mechanisms in order to demonstrate the working principle of $A E$ monitoring techniques for mooring systems. The work will also be extended in form of sea trials to study the practical feasibility of AE monitoring in noisy ocean environment.

\section{Acknowledgements}

JW is funded by the Natural Environment Research Council (NERC grant NE/L002434/1) as part of the GW4+ Doctoral Training Partnership (http://www.nercgw4plus.ac.uk/). IB is funded through the 
SuperGen UK Centre for Marine Energy Research (EPSRC grant EP/M014738/1). The use of the Ball hydrophone JS-B100-C4DS-PA, courtesy of SEA Ltd (formerly J+S Ltd) is also gratefully acknowledged.

\section{References}

[1] N. Healey, "Unbreakable - preventing mooring line failures," Oil \& Gas Agenda, 2014. [Online]. Available: http://www.growthmarkets-oil.com/features/featureunbreakable-preventingmooring-line-failures-4365180/. [Accessed: 23-Mar-2016].

[2] M. G. Brown, T. D. Hall, D. G. Marr, M. English, and R. O. Snell, "Floating production system, JIP FPS mooring integrity," Noble Denton Europe Limited, Aberdeeen, UK, 2006 [Online]. http://www.hse.gov.uk/research/rrpdf/rr444.pdf [Accessed: 08-May-2016].

[3] S. D. Weller, L. Johanning, P. Davies, and S. J. Banfield, "Synthetic mooring ropes for marine renewable energy applications," Renew. Energy, vol. 83, pp. 1268-1278, Nov. 2015.

[4] S. D. Weller, P. Davies, A. W. Vickers, and L. Johanning, "Synthetic rope responses in the context of load history: The influence of aging," Ocean Eng., vol. 96, pp. 192-204, Mar. 2015.

[5] Oil-Gas(UK), "Guidance on the Management of Ageing and Life Extension for UKCS Floating Production Installations," The UK Oil and Gas Industry Association Limited, London, UK, 2014.

[6] ABS-Consulting, "Study on Mooring System Integrity Management for Floating Structures," The Bureau of Safety and Environmental Enforcement, 2015 [Online]. http://www.bsee.gov/uploadedFiles/BSEE/Technology_and_Research/Technology_Assessme nt_Programs/Reports/700-799/730\%20AA.pdf [Accessed: 08-May-2016].

[7] Carbon Trust and Black \& Veatch, "Accelerating marine energy. The potential for cost reduction - insights from the Carbon Trust Marine Energy Accelerator," The Carbon Trust, London, UK, 2011. [Online]. Available: https://www.carbontrust.com/media/5675/ctc797.pdf [Accessed: 08-May-2016].

[8] Pulse-Structural-Monitoring, "Vessel, Platform and Mooring." [Online]. Available: http://www.pulse-monitoring.com/products-and-services-4/vessel-platform-and-mooring66. [Accessed: 24-Mar-2016].

[9] C. Campman, R. Edwards, and W. Hennessy, "Review of floating production platform real-time integrity monitoring systems worldwide," Rio Oil \& Gas Conference Proceedings, 24 pp., Rio de Janeiro, 2010.

[10] Tritech-RAMS, "Real - Time Continuous Integrity Monitoring of FPSO Mooring Lines \& Risers Using Multibeam Sonar Technology." [Online]. Available:

http://www.subseauk.com/documents/presentations/angus_lugsdin_-_tritech__tritech_rams.pdf [Accessed: 04-April-2016].

[11] N. F. Casey and P. A. A. Laura, "A review of the acoustic-emission monitoring of wire rope," Ocean Eng., vol. 24, no. 10, pp. 935-947, Nov. 1997.

[12] G. Drummond, J. F. Watson, and P. P. Acarnley, "Acoustic emission from wire ropes during proof load and fatigue testing," NDT E Int., vol. 40, no. 1, pp. 94-101, Jan. 2007.

[13] J. Walsh, I. Bashir, J. K. Garrett, P. R. Thies, P. Blondel, and L. Johanning, "Monitoring the condition of Marine Renewable Energy Devices through underwater Acoustic Emissions: Case study of a Wave Energy Converter in Falmouth Bay, UK," Renew. Energy, vol. 102, pp. 205-213, 2017.

[14] G. M. Wenz, "Acoustic Ambient Noise in the Ocean: Spectra and Sources," J. Acoust. Soc. Am., vol. 34, no. 12, pp. 1936-1956, Dec. 1962.

[15] L. Johanning, G. H. Smith, and J. Wolfram, "Mooring design approach for wave energy 
converters," Proc. Inst. Mech. Eng. Part M J. Eng. Marit. Environ., vol. 220, no. 4, pp. 159-174, Jan. 2006.

[16] S. D. Weller, P. Davies, A. W. Vickers, and L. Johanning, "Synthetic rope responses in the context of load history: Operational performance," Ocean Eng., vol. 83, pp. 111-124, Jun. 2014.

[17] Bridon "12 Strand roundline polyester rope, Fishing and Marine - Ship mooring, Bridon." [Online]. Available: http://www.bridon.com/uk/fishing-and-marine-ropes/ship-mooring/shipmooring-ropes/12-strand-roundline-polyester/. [Accessed: 20-Apr-2016].

[18] P. R. Thies, L. Johanning, K. A. Karikari-Boateng, C. Ng, and P. McKeever, "Component reliability test approaches for marine renewable energy," Proc. Inst. Mech. Eng. Part O J. Risk Reliab., vol. 229, no. 5, pp. 403-416, Apr. 2015.

[19] P. R. Thies, L. Johanning, I. Bashir, T. Tuk, M. Tuk, M. Marta, and S. Müller-Schütze, "Accelerated reliability testing of articulated cable bend restrictor for offshore wind applications," Int. J. Mar. Energy, vol. 16, pp. 65-82, 2016.

[20] J. L. and L. A. Rodríguez A., Weller S.D., Canedo J., Rodríguez R., González de Lena V., Thies P.R., Parish D., "Performance Comparison of Marine Renewable Energy Converter Mooring Lines Subjected to Real Sea and Accelerated Loads," in 11th European Wave and Tidal Energy Conference, 2015.

[21] S. Conforto and T. D'Alessio, "Spectral analysis for non-stationary signals from mechanical mesurements: A parametric approach," Mech. Syst. Signal Process., vol. 13, no. 3, pp. 395411, May 1999.

[22] M. Ainslie, Principles of Sonar Performance Modelling. Berlin, Heidelberg: Springer Berlin Heidelberg, 2010.

[23] G. G. R. Francois R. E., "Sound absorption based on ocean measurements: Part I:Pure water and magnesium sulfate contributions," J. Acoust. Soc. Am., vol. 72, no. 3, pp. 896-907, 1982. 